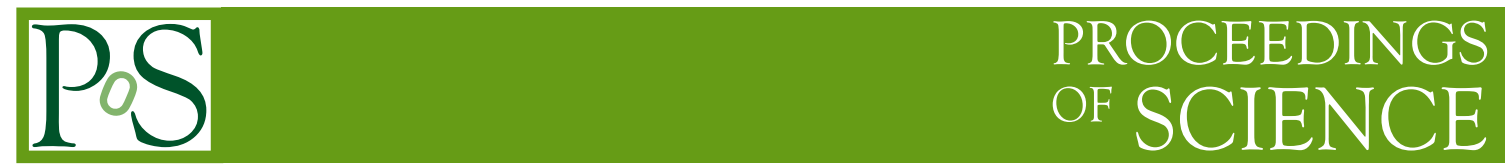

\title{
Tracking in 4 Dimensions
}

\section{N. Cartiglia*}

INFN Torino

E-mail: cartiglia@to.infn.it

F. Cenna, M. Ferrero, V. Monaco, M. Obertino, R. Sacchi

Università di Torino - INFN Torino

\section{G.F Dalla Betta, L. Pancheri}

Università di Trento

\section{Boscardin, G. Paternoster}

Fondazione Bruno Kessler

\section{G. Dellacasa, S. Garbolino, A. Rivetti, M. Rolo,V. Sola, A. Staiano} INFN, Torino

\section{R. Arcidiacono}

Università Piemonte Orientale - INFN Torino

\section{B. Baldassarri}

\section{UCL - INFN, Torino}

In this contribution I will review the progresses toward the construction of a tracking system able to measure the passage of charge particles with a combined precision of $\sim 10 \mathrm{ps}$ and $\sim 10$ $\mu \mathrm{m}$ either using a single type of sensor, able to concurrently measure position and time, or a combination of position and time sensors.

INFN Workshop on Future Detectors for HL-LHC

16-18 December, 2015

Aula Magna della Cavallerizza Reale, Torino, Italy

\footnotetext{
* Speaker.
} 


\section{The effect of timing information}

The inclusion of timing information in the structure of a recorded event has the capability of changing the way we design experiments, as this added dimension dramatically improves the reconstruction process. The most obvious simplification is that only time-compatible hits are used in the pattern recognition phase, discarding those hits that cannot be associated to a track due to an excessive time difference.

Depending on the type of sensors that will be used, timing information can be available at different stages in the reconstruction of an event. The most complete option is that timing is associated to each point of the track: in this case the electronics needs to be able to accurately measure the time of the hit in each pixel. This option is indeed quite difficult to achieve, due to the massive increase of power consumption that such a circuit would require. Nevertheless, as the potential gain that this option offers in terms of performances is the largest, we have set this option as our final goal. Figure 1 schematically shows the effect of having timing information for each hit.

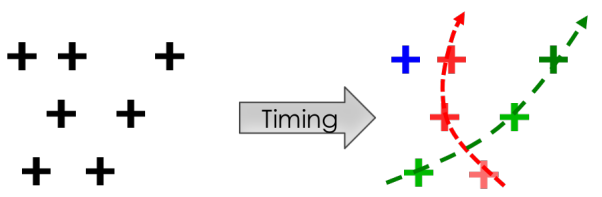

Figure 1: Effect of the inclusion of timing information at the hit level: only time-compatible points are associated to a track.

A very interesting option to deal with the increased power consumption mentioned above it is to reduce the granularity of the timing information, moving the timing sensors outside the volume of the tracker. This specific idea has been explored in order to improve Level 1 trigger decision, as timing information can be obtained faster than a full tracking reconstruction. Figure 2 shows an example of the added value of timing information at the trigger level: on the left, the situation without timing information indicates a 3-jet event, while on the right the 3 jets are correctly assigned to a 2-jet plus missing $E_{T}$ event plus an overlapping jet from another event.

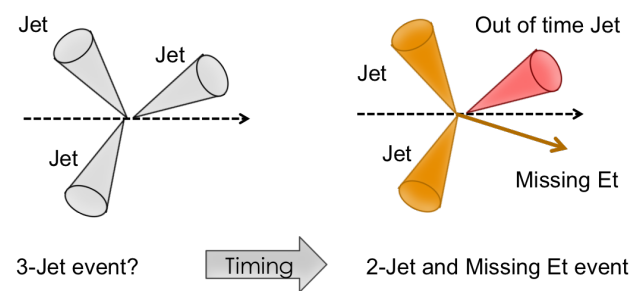

Figure 2: Effect of timing information of the topology of the event: a 3-jet event is re-interpreted as a 2-jet plus missing $E_{T}$ event plus an overlapping jet from different event.

To obtain the full power of timing information at the event reconstruction level, i.e. to maximize the precision of the reconstructed kinematical quantities, it is actually enough to assign a time to each track. This path is much easier than assigning time to each hit, as it can be done with a 
single dedicated timing layer either inside or outside the tracker volume. This path is now under consideration in the CMS upgrade, as it would retain a large part of the benefits of 4-D reconstruction, without the burden of redesigning the tracker system. Figure 3 shows schematically 3 situations where the timing information is crucial to the correct definition of the event topology: a) two overlapping events are recognized as one event with missing $E_{T}$ plus a minimum bias event, b) in $H \rightarrow \gamma \gamma$ events, the timing of the two photons identify a small region of space where the correct vertex is located: vertex timing allows making the correct match, c) secondary tracks can be correctly assigned to their vertex.

a)

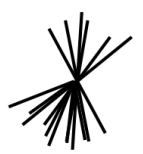

b)

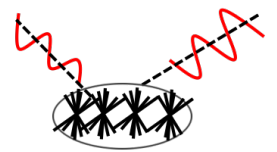

c)

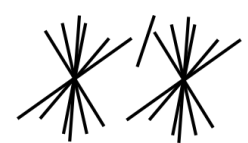

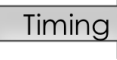
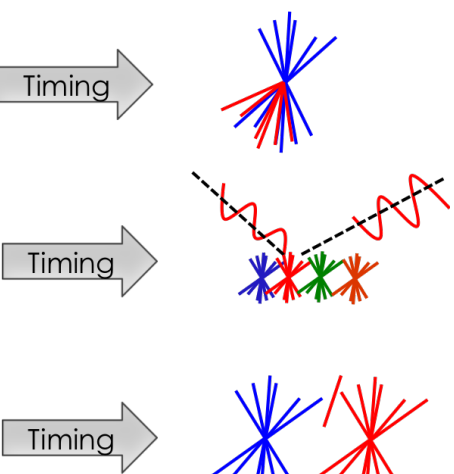

Figure 3: The knowledge of track-timing allows assigning correctly each track to the proper vertex: a) overlapping events, b) matching of the $H \rightarrow \gamma \gamma$ vertex, c) heavy flavor decays.

Considering a specific situation, at HL-LHC the number of events per bunch crossing will be of the order of 150-200, with an average distance between vertexes of 500 micron and a timing rms spread of 150 ps. Considering a vertex separation resolution of 250-300 micron along the beam direction (present resolution for CMS and ATLAS), there will be 10-15\% of vertexes composed by two events. This overlap will cause a degradation in the precision of the reconstructed variables, and lead to loss of events. We can therefore conclude that timing information at HL-LHC is equivalent of having additional luminosity.

\section{Time-tagging detectors}

Figure 4 shows the main components of a time-tagging detector. For a review of current trends in electronics see for example [1]. The sensor, shown as a capacitor with a current source in parallel, is read-out by a pre-amplifier that shapes the signal. The shaper's output is then compared to a fixed threshold to determine the time of arrival. In the following we will use this simplified model to explore the timing capabilities of various detectors, while for the moment we will not consider more complex and space-consuming approaches such as waveform sampling.

The time resolution $\sigma_{t}$ can be expressed as the sum of several terms: (i) Jitter, (ii) Landau shape variation, (iii) Time Walk, (iv) signal distortion, and (v) TDC binning:

$$
\sigma_{t}^{2}=\sigma_{\text {Jitter }}^{2}+\sigma_{\text {Landau Noise }}^{2}+\sigma_{\text {Distortion }}^{2}+\sigma_{\text {Time Walk }}^{2}+\sigma_{T D C}^{2} .
$$

For the sake of clarity, in the following we will assume two simplifications: 


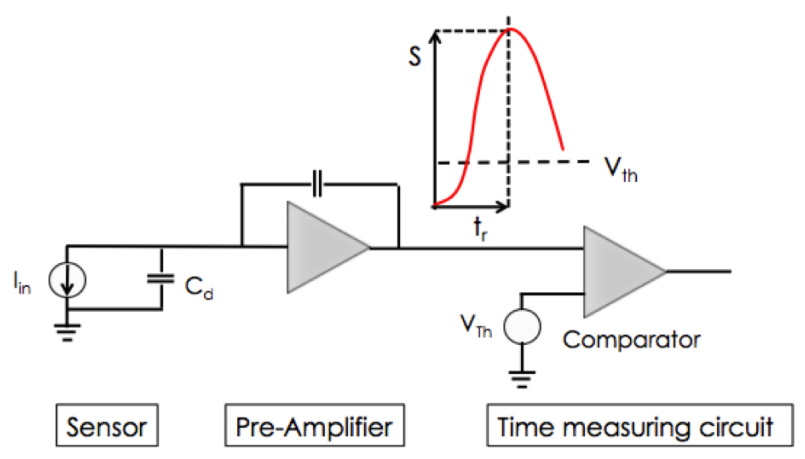

Figure 4: Main components of a time-tagging detectors. The time is measured when the signal crosses the threshold.

- we consider the effect of time walk (see [2] for details) compensated by an appropriate electronic circuit (either Constant Fraction Discriminator or Time over Threshold), see Section 4.1. With this assumption, the effect of Landau variations in signal amplitude are compensated, but not that of shape variation (in eq. 2.1 this second contribution is indicated as Landau noise, $\sigma_{\text {Landau Noise }}^{2}$.

- the contribution of TDC binning to be below $10 \mathrm{ps}$ and therefore it will be ignored.

\subsection{Jitter}

The jitter term represents the time uncertainty caused by the early or late firing of the comparator due to the presence of noise on the signal, it is directly proportional to the noise $N$ of the system and it is inversely proportional to the slope of the signal around the value of the comparator threshold, Figure 5 a). Assuming a constant slope we can write $d V / d t=S / t_{r}$ and therefore:

$$
\sigma_{J}=\frac{N}{d V / d t}=\frac{t_{r}}{S / N}
$$

\subsection{The effect Landau fluctuations: time walk and Landau noise}

The final limit to signal uniformity is given by the physics governing energy deposition: the charge distribution created by an ionizing particle crossing a sensor varies on an event-by-event basis. These variations not only produce an overall change in signal magnitude, which is at the root of the time walk effect (that we assumed perfectly corrected by electronics), but also produce a more irregular current signal (Landau noise). The top part in Figure 5 b) shows 3 examples of the simulated [3] energy deposition of a minimum ionizing particle, while the bottom part the associated generated current signals and their components. As the picture shows, the variations are rather large and they can severely degrade the achievable time resolution. There are two ways to mitigate this effect: (i) integrating the output current over times longer than the typical spike length and (ii) using thin sensors, as their steeper signal is more immune to signal fluctuations. 


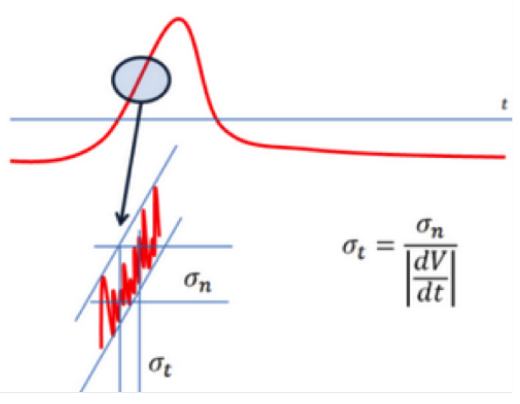

a)

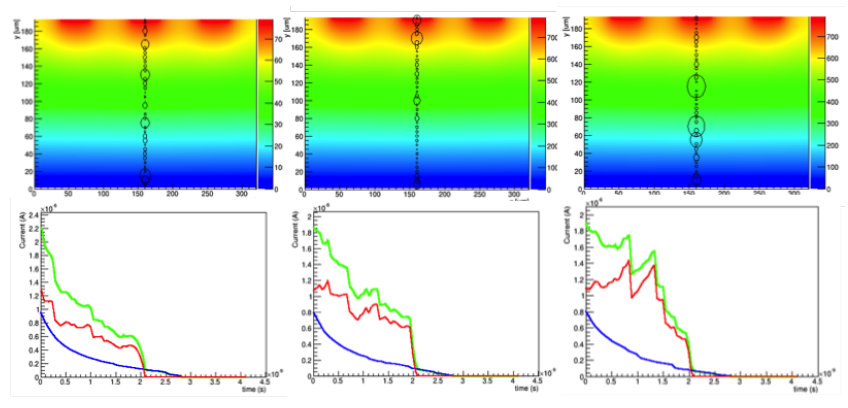

b)

Figure 5: a) Time Jitter, b) Energy deposits in a silicon detectors and the corresponding current signals.

\subsection{Signal Distortion: non uniform weighting field and not saturated drift velocity}

In every particle detector, the shape of the induced current signal can be calculated using Ramo's [4] theorem that states that the current induced by a charge carrier is proportional to its electric charge $q$, the drift velocity $v$ and the weighting field $E_{w}$, equation (2.3):

$$
i \propto q v E_{w} .
$$

This equation indicates several key points in the design of sensors for accurate timing, shown in Figure 6:

- the drift velocity needs to be constant throughout the volume of the sensor. Non-uniform drift velocity induce variations in signal shape as a function of the hit position, Figure 6 a), spoiling the overall time resolution. The easiest way to obtain uniform drift velocity throughout the sensor is to have an electric field high enough to move the carriers with saturated drift velocity.

- the weighting field $E_{w}$ represents the capacitive coupling of a charge $e$ with the read-out electrode. If this coupling depends on the impinging particle position along the strip pitch, Figure $6 \mathrm{~b}$ ), the signal shape would be different depending on the hit position, spoiling the time resolution. Strips need to have the width very similar to the pitch, and larger than the signal thickness: width $\sim$ pitch $»$ thickness.

\subsection{Optimum Sensor Design}

Combining the information presented above, it is clear that not all geometries can be used in time tagging detectors. The most important points to have a detector with excellent time resolution are:

- High field to have saturated velocity.

- Geometries as similar as possible to parallel plate capacitors, offering uniform electric and weighting fields 


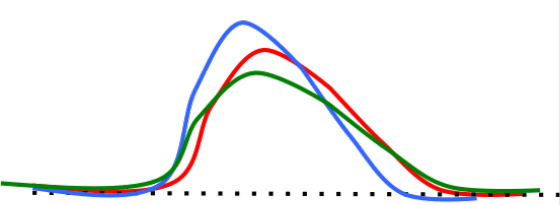

a)

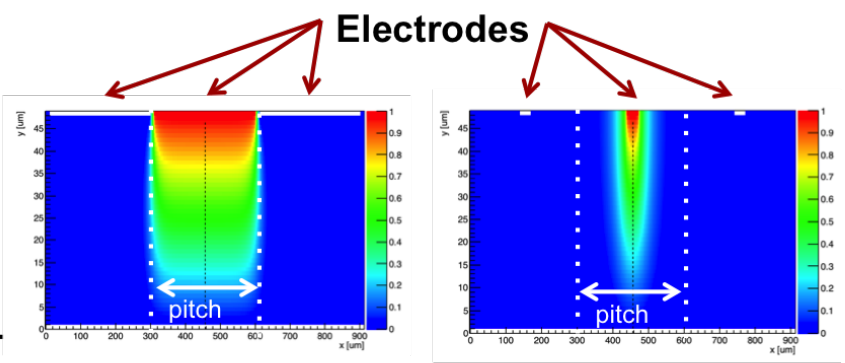

Thin Strip

b)

Figure 6: a) Effect of velocity variation on the signal shape b) Weighting field for two configurations: (left) Wide strips, (right) Thin strips.

- High velocity carriers, to have large $d V / d t$

- Low capacitance, to minimize jitter

- Small volumes, to minimize leakage current and therefore Shot noise

\subsection{Roadmap}

The integration of time-tagging capabilities into a position sensor produces a steep increase in system complexity. Part of this complexity can be addressed by very smart architectures, new technological nodes (for example $65 \mathrm{~nm}$ ) allowing higher circuit densities and new chip designs [5]. However the present bottlenecks inherent to hybrid systems, having sensors and electronics built on separated substrates, will ultimately limit the complexity and drive the cost. Most likely the real turning point of 4D tracking will happen when monolithic technology will be mature enough to allow integrating the sensor and the electronics in the same substrate, reducing interconnections and keeping the capacitance of each sensor low. As we are deciding now (2016) the choices for HL-LHC (2025), this evolution will not be used at Hl-LHC, but it will probably appear first in smaller experiments, and then be used on larger scales.

\section{Possible Detector for a 4D tracker}

If we assume that a 4D tracker should maintain the key aspects of current 3D tracker designs, then the type of possible detectors is limited by material budget consideration to semiconductor devices, mostly silicon and diamond. Assuming all other aspects being equal (capacitance, drift velocity, manufacturing capability, cost), the key quantity to be minimized by every detector is the jitter term expressed by equation eq.2.2. We will here briefly review 4 possible approaches, outlining the strong and weak points of each one.

\subsection{Silicon detectors}

Standard silicon detectors can be used in timing applications, provided the sensor geometry is appropriate. Currently the NA62 experiment [6] is employing a track-timing detector, the so 
called GiGatracker, that uses 300-micron thick sensors with 300-micron pixels. The expected time resolution is around $\sigma_{t} \sim 150 \mathrm{ps}$. Recently [7], employing an extremely low noise new circuit, a resolution of $\sigma_{t} \sim 105 \mathrm{ps}$ has been reached using a 100-micron thick, $2 \mathrm{~mm}$ square pad sensor. Standard silicon sensors have therefore the capability of reaching good time resolutions, however it is rather difficult to reach resolutions better than $\sigma_{t} \sim 80-100 \mathrm{ps}$ given their small signal.

\subsection{Diamond detectors}

Diamond has a large energy gap, therefore it is very radiation resistance and it has almost no leakage current; it has a small dialectric constant, so the capacitance is low, and carriers have high mobility. The main drawback is the small signal amplitude (due to the large energy gap), and the limited commercial offer. Figure 7 shows two techniques to increase the signal amplitude: a) stacking sensors, and b) grazing.

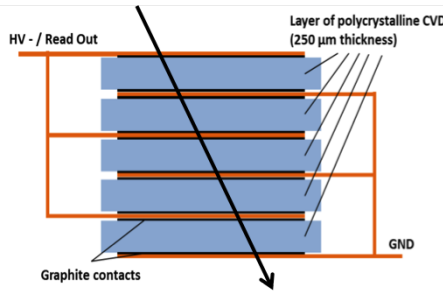

a)

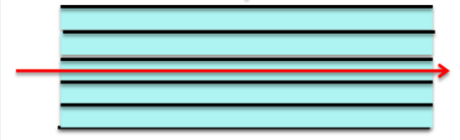

b)

Figure 7: Two configurations to increase the signal amplitude in diamond detectors: a) Stack b) Grazing

Diamond detectors have been therefore used successfully in time tagging sensors as they have low noise (due to their small capacitance), and a large $d V / d t$. The best result achieved so far has been a time resolution of $\sigma_{t} \sim 100 \mathrm{ps}$, obtained by the TOTEM collaboration. Given the difficulties of large scale production, diamond detectors are therefore ideally suited for small area sensors in high radiation environments.

\subsection{APD - Avalanche PhotoDiode}

APD can successfully be employed in the detection of charged particles. APD are normally quite thin, 30-50 micron, so the initial number of charge carriers generated by a MIP particles is rather small, 2-3000 e/h pairs, however their signal is very large since they have a gain of the order of 50 - 500. Given the short drift distance, the signal is very short and steep, with a very large $d V / d t$ : APD have therefore excellent time resolution, of the order of $\sigma_{t} \sim 30 \mathrm{ps}$. The same quality that makes APD very good timing sensors, high gain, is however also causing drawbacks: (i) when irradiated, APD have very high Shot noise (due to the multiplication of the leakage current, see section 5.3), (ii) they cannot be easily segmented, and (iii) they suffer from electric breakdown. APD are therefore a very good choice for single pad systems in low radiation environments.

\subsection{LGAD - Low-Gain Avalanche Detectors}

LGAD is a new concept in silicon detector design, merging the best characteristics of standard silicon sensors with the main feature of APDs. The overarching idea is to design silicon detectors 
with signals that are a factor of 10 higher than those of standard sensors, however without the problems connected with the APD high gain $[8,9,10,11,12]$.

Charge multiplication in silicon sensors happens when the charge carriers are in electric fields of the order of $E \sim 300 \mathrm{kV} / \mathrm{cm}$. Under this condition the electrons (and to less extent the holes) acquire sufficient kinetic energy that are able to generate additional e/h pairs. A field value of 300 $\mathrm{kV} / \mathrm{cm}$ is not reachable applying an external voltage $V_{\text {Bias }}$ without causing electrical breakdown, but it is obtained by implanting an appropriate charge density that locally generates very high fields $\left(N_{D} \sim 10^{16} / \mathrm{cm}^{3}\right)$. The gain has an exponential dependence on the electric field $N(l)=N_{o} e^{\alpha(E) l}$, where $\alpha(E)$ is a strong function of the electric field and $l$ is the path length inside the high field region. The additional doping layer present at the $n-p$ junction in the LGAD design, Figure 8, generates the high field necessary to achieve charge multiplication.

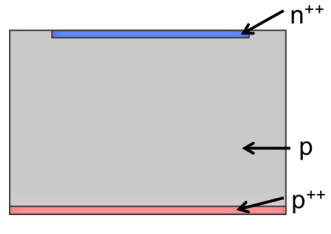

Traditional Silicon Diode

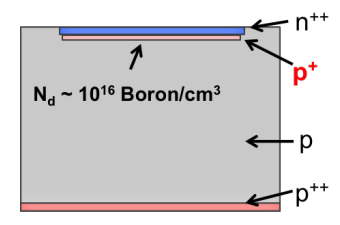

Low Gain Avalanche Diode

Figure 8: Schematic of a traditional silicon diode (left) and of a Low-Gain Avalanche Diode (right).

We have developed a full simulation program, WF2 [3], to study the property of signal formation in silicon detectors and the effect of gain. According to WF2, LGAD have the potentiality of replacing standard silicon sensors in almost every application, with the added advantage of having a large signal $d V / d t$ and therefore being able to measure time accurately. In the following, we will use the name of "Ultra-Fast Silicon detectors" (UFSD) to indicate LGAD sensors optimized for timing performances.

\section{Electronics for time tagging detectors}

The pre-amplifiers suited for time-tagging detectors can be divided in two large categories: (i) current amplifiers (CA) or (ii) charge sensitive amplifiers (CSA). With CA the signals are amplified without strong additional shaping while with CSA the signals are integrated and shaped. There are several issues that need to be considered when using either approach: CA are much faster, and they are able to take full advantage of the very fast signal slew rate but they have a higher noise, while CSA are somewhat slower but the integration they perform makes the output signal more immune to noise and Landau fluctuations. The choice between these two architectures needs to be evaluated in conjunction with the sensor dimensions since if the unavoidable signal integration due to the detector capacitance is enough to smooth out the effect of Landau fluctuations, then CA will provide the best results while if this is not the case then the second integration offered by the CSA is needed. 


\subsection{Choice of Time-walk correction circuits}

Time-walk, the unavoidable process by which larger signals cross a given threshold earlier than smaller ones, needs to be corrected by an appropriate electronic circuit. The three most common solutions are illustrated in Figure 9: (a) Constant Fraction Discriminator (CFD), which sets the time of arrival of a particle when the signal reaches a given fraction of the total amplitude, (b) Time over Threshold (ToT), that uses two time points to evaluated the amplitude of the signal, and apply a correction amplitude-dependent to the first time point $t_{1}$ and (c) Multiple Samplings (MS), where the signal is sampled multiple times, and a fit is used to define the particle time. CFD and ToT are simpler solutions, and they can be implemented per pixel within the read-out chip. MS is instead a rather complex algorithm as it requires the full digitization of the signal: this solution gives the best performance, but it can be used only for systems with a limited number of pixels as it needs a fair amount of computing power. The choice among these 3 possibilities is a system decision that needs to be taken considering all other aspects of the experiment.
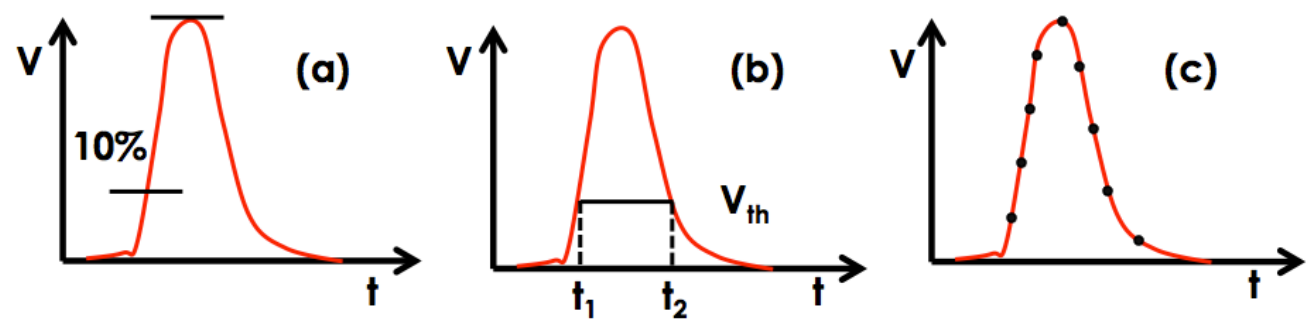

Figure 9: Time-walk correction techniques: (a) Constant fraction Discriminator, (b) Time Over Threshold, (c) Multiple Samplings.

\subsection{Interplay of signal rise time, detector capacitance and read-out input impedance}

The charges collected on the read-out electrode of the sensor move to the input of the read-out electronics with a time constant $\tau$ given by the product of the detector capacitance $C_{d e t}$ and the read-out input impedance $R_{\text {in }}: \tau=R_{\text {in }} C_{\text {det }}$, Figure 10 .

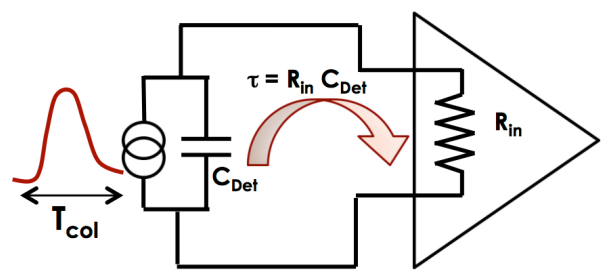

Figure 10: Interplay of the signal rise time, detector capacitance and read-out input impedance.

In order not to slow down the signal rise time, $\tau$ has to be shorter or, at most, of the same order of the signal current rise time, $t_{C u r}$. This constrain is strongly linking sensor and electronics designs, as the electronics should be such that it does not slow down very fast input signals. For example, pre-amplifiers that use SiGe technologies tend to have higher input impedance (100-300 
$\mathrm{Ohm})$ and therefore can be coupled only to small sensors $\left(C_{D e t}<2 \mathrm{pF}\right)$, so that the value of $\tau$ remains below $t_{C u r}\left(t_{C u r} \sim 500\right.$ ps for a $50 \mu \mathrm{m}$ thick sensor $)$.

\subsection{Amplifier rise time}

The signal rise time at the output of the pre-amplifier determines $d V / d t$ and therefore is of the utmost importance in time-tagging detectors. We can identify 3 distinct effects that influence the signal slew rate, eq. 4.1: (i) the signal rise time $t_{C u r}$ that depends on the properties of the sensor, (ii) the $t_{R C}=R_{i n} C_{d e t}$ time constant, as explained in section 4.2, and (iii) the amplifier rise time $t_{A m p}$, which should be matched to $t_{C u r}$ and $t_{R C}$ to minimize the noise of the amplifier:

$$
t_{\text {rise }}=\sqrt{t_{\text {Cur }}^{2}+t_{R C}^{2}+t_{\text {Amp }}^{2}}
$$

It can be demonstrated that the condition $t_{A m p}^{2} \sim t_{C u r}^{2}+t_{R C}^{2}$ minimize the noise contribution while affecting the slew rate minimally.

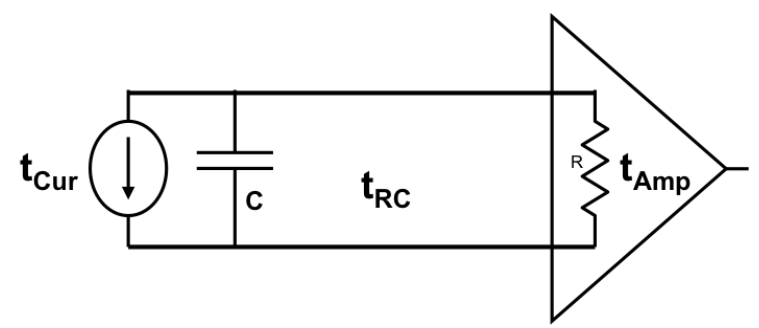

Figure 11: Schematic representation of the 3 main time constants affecting the signal rise time at the preamplifier output.

\section{Design issues for a 4D tracker using UFSD}

In this section we present further studies on how a 4D tracker can be designed using Ultra-fast silicon detectors.

\subsection{The effect of charge multiplication}

Using WF2 we can simulate the output signal of UFSD sensors as a function of many parameters, such as the gain value, sensor thickness, electrode segmentation, and external electric field. Figure 12 shows the simulated current, and its components, for a 50-micron thick detector. The initial electrons (red), drifting toward the $\mathrm{n}++$ electrode, go through the gain layer and generate additional e/h pairs. The gain electrons (violet) are readily absorbed by the cathode while the gain holes (light blue) drift toward the anode and they generate a large current.

The gain dramatically increases the signal amplitude, producing a much higher slew rate. The value of the current generated by a gain $G$ can be estimated in the following way: (i) in a given time interval $d t$, the number of electrons entering the gain region is $75 v d t$ (assuming $75 \mathrm{e} / \mathrm{h}$ pairs per micron); and (ii) these electrons generate $d N_{\text {Gain }} \propto 75 \mathrm{v} d t G$ new e/h pairs. Using again Ramo's theorem, the current induced by these new charges is given by:

$$
d i_{\text {Gain }}=d N_{\text {Gain }} q v_{\text {sat }} \frac{k}{d} \propto \frac{G}{d} d t,
$$




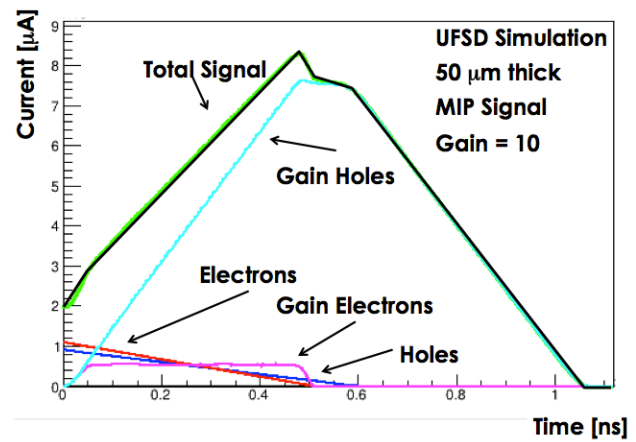

Figure 12: UFSD simulated current signal for a 50-micron thick detector.

which leads to the following expression for the slew rate:

$$
\frac{d i_{\text {Gain }}}{d t} \sim \frac{d V}{d t} \propto \frac{G}{d} .
$$

Equation (5.2) demonstrates a very important feature of UFSD: the slew rate increase due to the gain mechanism is proportional to the ratio of the gain value over the sensor thickness $(G / d)$, therefore thin detectors with high gain provide the best time resolution. Specifically, the maximum signal amplitude is controlled only by the gain value, while the signal rise time only by the sensor thickness, Figure 13.

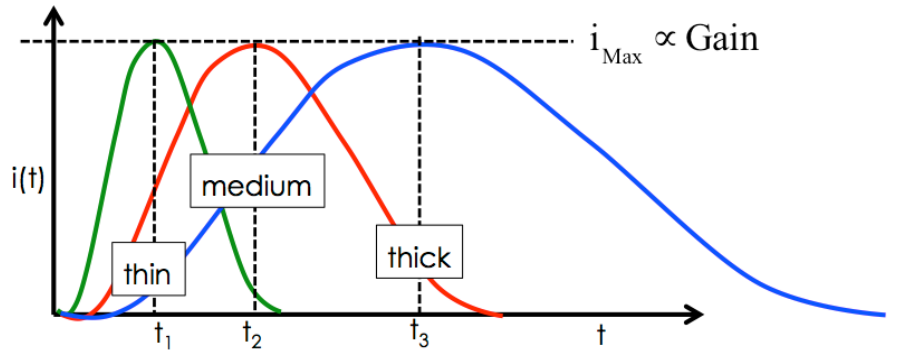

Figure 13: In UFSD the maximum signal amplitude depends only on the gain value, while the signal rise time only on the sensor thickness: sensors of 3 different thicknesses (thin, medium, thick) with the same gain have signals with the same amplitude but with different rise time.

Using WF2 we have cross-checked this prediction simulating the slew rate for different sensors thicknesses and gains, Figure 14: the slew rate in thick sensors, 200- and 300-micron, is a factor of $\sim 2$ steeper than that of traditional sensors, while in thin detectors, 50- and 100-micron thick, the slew rate is 5-6 times steeper.

\subsection{Landau noise in UFSD sensors}

With WF2 we have studied in details the effect of Landau noise on time resolution, Figure 15. The picture shows several important effects: (i) Landau noise sets a physical limit to the precision of a given sensor which is of the order of $20 \mathrm{ps}$ in thin sensors, and much larger for thicker sensors, (ii) Landau noise is minimized by setting the comparator threshold as low as possible, (iii) thin detectors are less prone to Landau noise. 


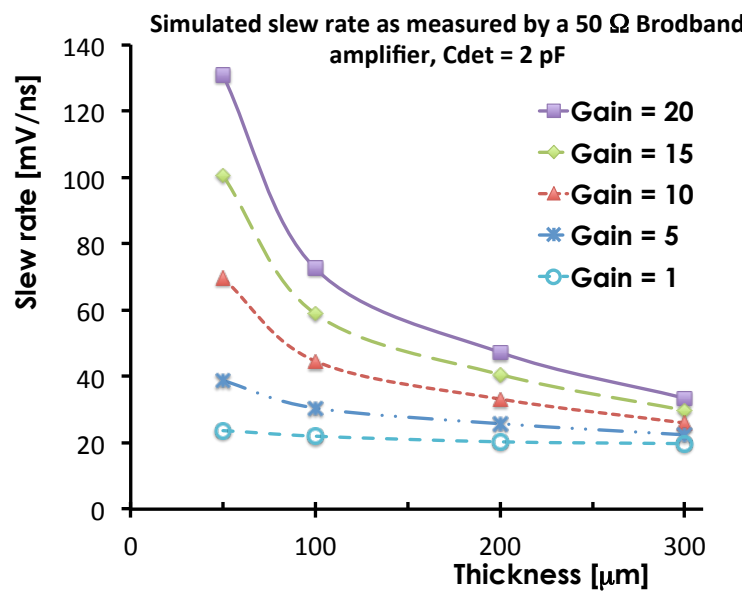

Figure 14: Simulated UFSD slew rate as a function of gain and sensor thickness. Thin sensors with even moderate gain (10-20) achieve a much higher slew rate than traditional sensors (gain $=1)$.

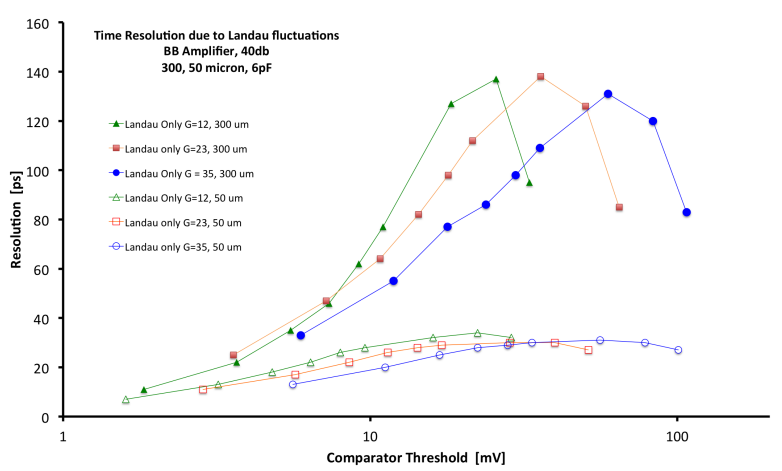

Figure 15: Effect of Landau noise on time resolution. Best results are obtained for thin sensors, while the effect of gain on Landau noise is less important.

\subsection{Shot Noise in UFSD sensors}

Shot noise arises when charge carriers cross a potential barrier, as it happens in silicon sensors. In sensors such as UFSD or APD this effect is enhanced by the gain and for this reason Shot noise can be the dominant source of noise for this type of detectors. As shown in Figure 16 a), the sensor leakage current is the sum of two components: (i) surface current, that does go through the multiplication layer, and (ii) bulk current, that is multiplied by the gain mechanism.

When carriers undergo multiplication, there is an additional mechanism that enhance Shot noise: multiplication is a statistical process, therefore some carries multiply more than others, causing an increase in noise, the so called excess noise factor, ENF. This effect is in addition to the increase in noise due to the gain value that simply multiply the leakage current. ENF causes a very peculiar effect: in device with gain, as the gain increases the ratio signal/noise $(\mathrm{S} / \mathrm{N})$ becomes 


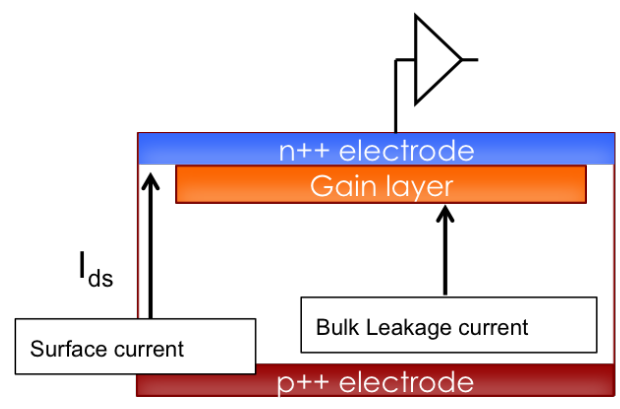

a)

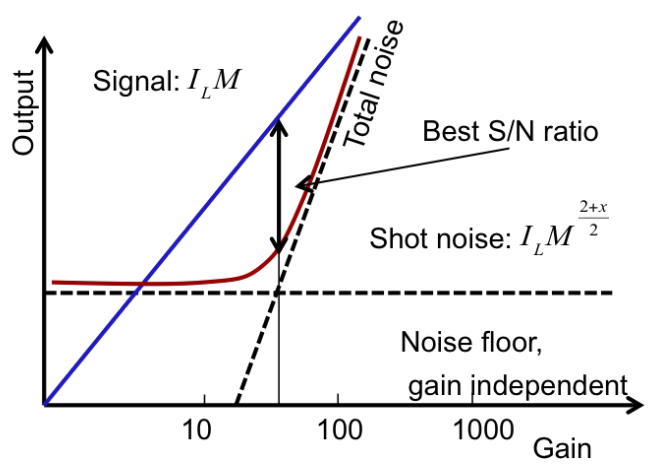

b)

Figure 16: a) Sketch of the Shot noise mechanism in sensors with internal gain: bulk current is multiplied by the gain, while surface current is not. b) For increasing gain, Shot noise increases faster than the signal.

smaller since Shot noise increases faster than the signal, Figure $16 \mathrm{~b}$ ). In order to obtain a beneficial effect from the gain mechanism is therefore necessary to have a gain value small enough (gain $\leq$ 20) that the signal increases while the noise increment is small enough to be in the shadow of the electronic noise floor.

The Shot noise current density is given by:

$$
i_{\text {Shot }}^{2}=2 e I_{\text {Det }}=2 e\left[I_{\text {Surface }}+I_{\text {Bulk }} M^{2} M^{x}\right]
$$

where $M$ is the gain value, and $x$ is the excess noise index. Integrating over the electronic frequency spectrum we obtain the Shot noise equivalent noise charge (ENC) [1]:

$$
E N C_{\text {Shot }}=\sqrt{\frac{I_{\text {Bulk }} M^{2} M^{x}}{2 e} \tau}
$$

where $\tau$ is the amplifier integration time.

Shot noise is normally smaller than the electronic noise floor for un-irradiated sensors, but it can become the dominant source of noise for irradiated detectors. Figure 17 shows the value of Shot noise for a $4 \mathrm{~mm}^{2}$ 50-micron thick silicon sensor, assuming a 2-ns long integration time. In the plots the electronic noise is assumed to be 450 ENC. Figure 17 a) demonstrates the dramatic effect of gain on Shot noise, while Figure $17 \mathrm{~b}$ ) the effect of temperature (leakage current decreases a factor of 2 every 7 degrees).

Figure 17 demonstrates that Shot noise can become the most important source of noise for irradiated sensors with gain, and suggests that low gain and low temperature can keep this effect under control. Additionally, Shot noise can be kept under control by keeping small the volume of the each pixel/strip, as leakage current depends on the overall volume: $I_{\text {Leak }}=\alpha * \Phi * V$, with $\alpha=3 \cdot 10^{-17} \mathrm{~cm}^{-1}$ and $\Phi$ the particle fluence in $n_{e q} / \mathrm{cm}^{2}$.

\subsection{Irradiation effect in UFSD sensors}

Radiation damage causes three main effects: 


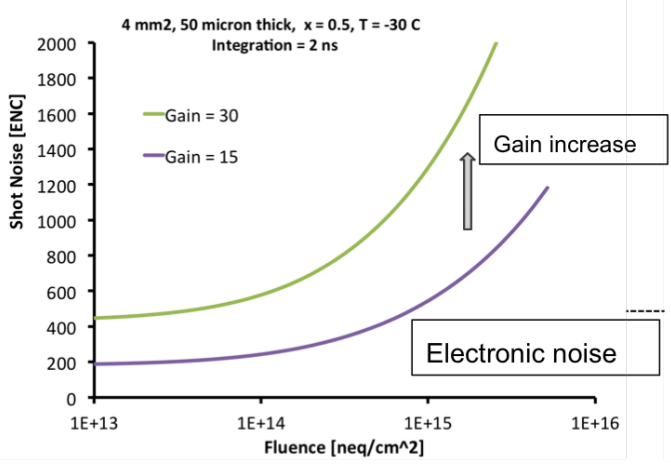

a)

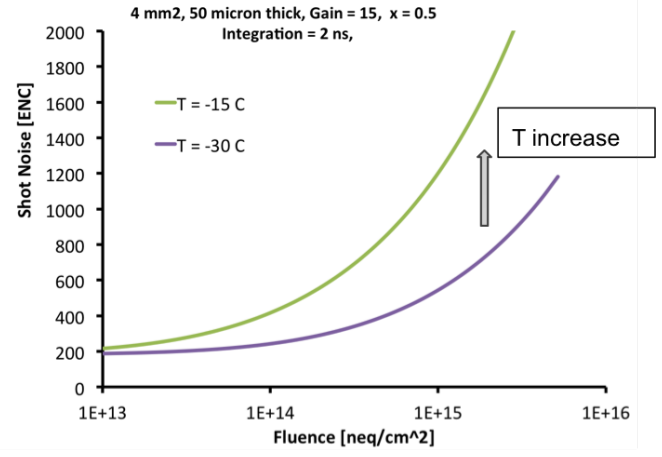

b)

Figure 17: a) Shot noise increase as a function of fluence for two different gain values. b) Shot noise increase as a function of fluence for two different temperature values

- Decrease of charge collection efficiency

- Increase of leakage current

- Changes in doping concentration

Decrease of charge collection efficiency: Charge collection efficiency (CCE) measures the fraction of charge carriers that are not trapped by lattice defects. This fraction decreases with increasing radiation levels (with differences depending on the type of silicon and type of irradiation) and with increasing drift length. As a rule of thumb, for irradiation levels around $10^{15} n_{e q} / \mathrm{cm}^{2}$, the mean free path in silicon is around 50 micron. Figure 18 shows the signal changes as a function of radiation level for a 50-micron thick sensor: the effect is rather small up to a fluency of $10^{15} n_{e q} / \mathrm{cm}^{2}$, and it becomes important above a fluency of $5 \cdot 10^{15} n_{e q} / \mathrm{cm}^{2}$. Interestingly, the initial edge of the signal, used for timing, does not change much.

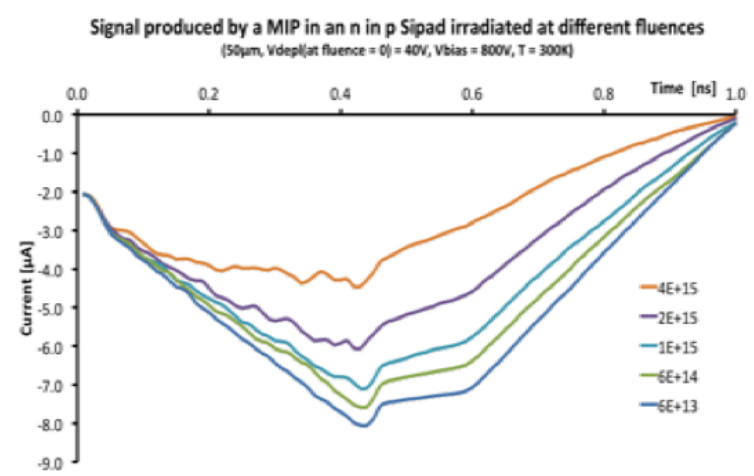

Figure 18: Signal change in a 50-micron silicon sensor as a function of irradiation levels (the only effect considered in this plot is charge trapping). 
Increase of leakage current: An increase in leakage current causes two important effects: (i) a larger noise, as explained in Section 5.3, and (ii) a change in the apparent doping concentration. This second effect can be quite important, and it is presently under intense study.

Changes in doping concentration: UFSD sensors have shown a decrease of gain values for fluences above $10^{-14} n_{e q} / \mathrm{cm}^{2}$, with a complete disappearance of the gain at $10^{-14} n_{e q} / \mathrm{cm}^{2}$. This effect has not been understood yet, but there are two possible explanations: (i) an inactivation of acceptors due to radiation defects [13], and (ii) a dynamic reduction of the gain layer doping due to charge trapping.

Radiation damage causes several intertwined effects. First, it causes the doping of a silicon detector to become more "p-type", it is well know that n-type bulk becomes p-type bulk, the so called "type inversion". This change is due to defects that act as permanent acceptors. There is however a second effect that is also very important: there is experimental evidence [14], supported by simulation studies [15], that the leakage current fills traps in the bulk (i.e. electrons and holes are trapped in the defects of the bulk) changing the doping profile. Figure 19 illustrates this effect: on the left side, a), it shows the doping profile of a n-in-p silicon sensor, while on the right side, b), it shows how this profile is affected by dynamic charge trapping: the electrons drifting up are on average more trapped near the n-electrode, while the opposite it true for the holes. As "negatively trapped charges" are equivalent to p-doping, and "positively trapped charges" to n-doping, the dynamic effect of large leakage current is to create a double junction: n-p-n-p. Double junction effects distort the field and change the signal shape significantly.

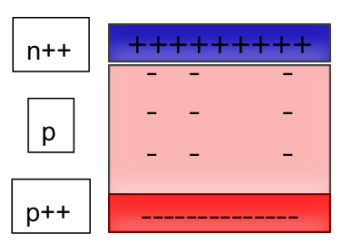

a)

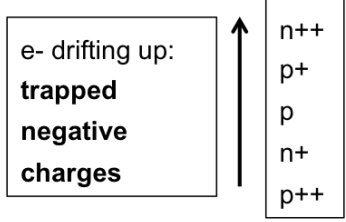

charges

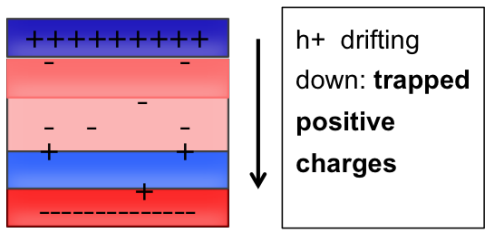

b)

Figure 19: a) n-in-p silicon sensor. b) As "negatively trapped charges" are equivalent to p-doping, and "positively trapped charges" to n-doping, the dynamic effect of large leakage current is to create a double junction: n-p-n-p

In sensors with gain, such as UFSD or APD, the effect of charge trapping can be very important due to the multiplication of the leakage current. Contrary to normal sensors, in the presence of gain the leakage current is dominated by the holes: the electrons multiply in the gain layer, but the gain electrons are immediately absorbed, while the gain holes are drifting toward the opposite side of the sensor, flooding the bulk with positive charges. Under this condition, the double-junction effect does not appear, as the trapped electrons are compensated by many more trapped holes, shown in Figure 20, and the sensor becomes more n-type. According to simulation, this shift in doping is enough to decrease the gain layer p-doping and reduce the gain value.

There are currently two research paths in the investigation of gain reduction, aiming at establishing a radiation hard design for UFSD: (i) change the dopant of the p-type gain layer from Boron to Gallium, as Gallium has been shown to be more radiation resistant, (ii) development of very thin sensors, to decrease the amount of leakage current and therefore trapping. This solution, together 


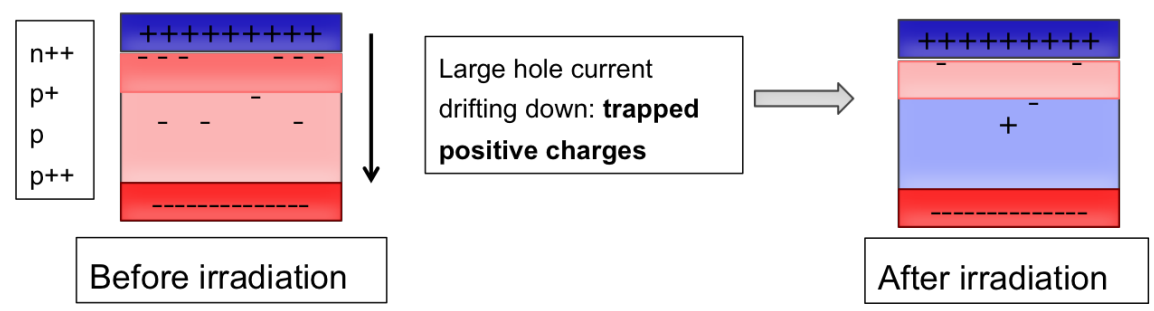

Figure 20: Dynamic effect of doping changes in sensors with gain. The sensor becomes more n-type, and the doping in the gain layer decreases, causing a decrease in gain value.

with operating the sensors at low temperature (-20 degrees) should reduce the amount of leakage current by at least a factor of 100 , which in turn will decrease the dynamic n-type creation.

\subsection{Testbeam results and extrapolation of UFSD performances}

We have performed several beam tests to validate the design of UFSD sensors. The results, together with simulation predictions, are shown in Figure 21. Best results for a 300-micron thick sensors are $\sigma_{t} \sim 120 \mathrm{ps}$, due equally to, according to simulation, jitter and Landau noise contributions. According to simulation, a dramatic improvement is predicted for thin sensors, reaching $\sigma_{t} \sim 30$ ps for 50-micron thick sensors.

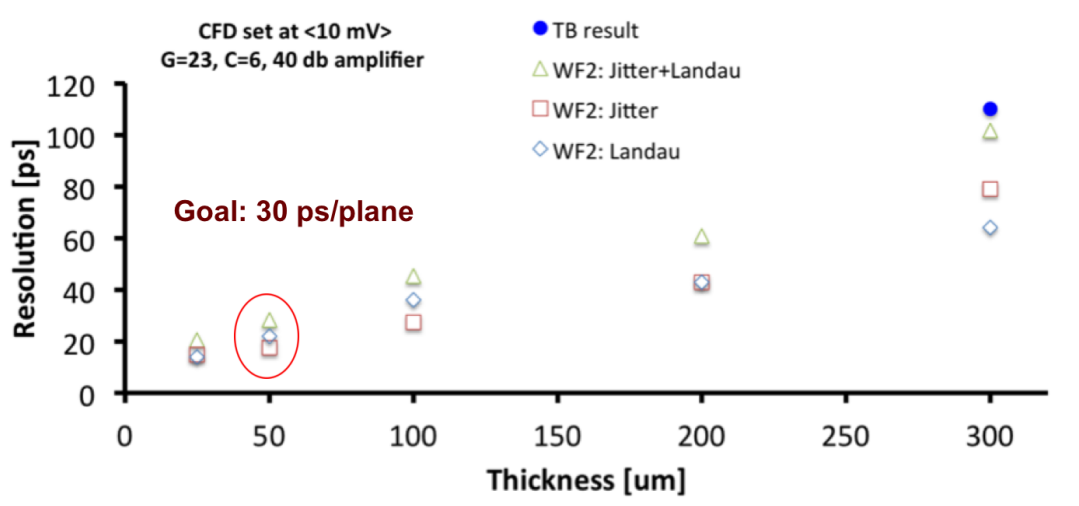

Figure 21: Beam test results for 300-micron UFSD sensors, and extrapolation to thin sensors. For each thickness, the predictions obtained by the WF2 program for the Landau noise and the jitter term are also shown.

\section{Conclusions and outlook}

Tracking in 4 dimensions requires the development of dedicated sensors and associated electronics. Several approaches are currently being investigated (Diamond, UFSD, APD, Silicon), and each one is responding to specific experimental requests. UFSD sensors have the capability of being used in large scale systems, as the have the same properties of normal silicon detectors, with the added features of larger signals. In order to have good and stable signals, necessary for timing 
applications, not all geometries are possible, therefore large-area detectors with timing capabilities will need to find the appropriate compromises in terms of timing and tracking capabilities.

\subsection{UFSD design}

The design of UFSD is based on two main points: low gain and thin sensors.

Low gain: needed to easily achieve segmentation, keep Shot noise under control, reduce dynamic doping

Thin sensors: maximize the slew rate, decrease the amount of leakage current, minimize Landau noise, easily achieve parallel plate geometries even with small pixels.

\section{References}

[1] A. Rivetti, et al., Electronics for Fast Tracking Detector, HSTD9, Hiroshima, Japan, NIM A765 (2014) 202-208

[2] N. Cartiglia et al., Design Optimization of Ultra-Fast Silicon Detector, NIM A96 (2015) 141-148

[3] F. Cenna et al, Weightfield2: A fast simulator for silicon and diamond solid state detector NIM A796 (2015) 149-153.

[4] S. Ramo, "Currents Induced by Electron Motion". Proceedings of the IRE 27 (9): 584Đ585. doi:10.1109/JRPROC.1939.228757

[5] See talk by A. Rivetti, this proceeding

[6] E Martin et al., Review of results for the NA62 Gigatracker read-out prototype jinst72012C03030

[7] M. Benoit et al., http://arxiv.org/abs/1511.04231

[8] P. Fernandez, et al., Simulation of new p-type strip detectors with trench to enhance the charge multiplication effect in the n-type electrodes, NIM A658 (2011) 98-102 .

[9] G. Pellegrini et al., Technology developments and first measurements of Low Gain Avalanche Detectors (LGAD) for High Energy Physics applications, NIM A765 (2014) 12-16

[10] G-F Dalla Betta et al, Design and TCAD simulation of double-sided pixelated low gain avalanche detectors, NIM A796 (2015) 154-157

[11] H.-W. Sadrozinski et al., Sensors for ultra-fast silicon detectors, NIM A765 (2014) 7-11.

[12] H.-W. Sadrozinski et al., Ultra-fast silicon detectors, NIM A730 (2013) 226-231.

[13] G. Kramberger et al., Radiation effects in Low Gain Avalanche Detectors after hadron irradiations, JINST, 10 - 07 (2015), P07006

[14] V. Chiochia et al., A double junction model of irradiated silicon pixel sensors for LHCNIM A568 (2006) 51-55

[15] RD50 collaboration,http://rd50.web.cern.ch/rd50/. 